\title{
Propos liminaire pour introduire sans trop induire
}

\section{Hugues Hotier}

\section{OpenEdition}

Journals

Édition électronique

URL : http://journals.openedition.org/communicationorganisation/1978

DOI : 10.4000/communicationorganisation. 1978

ISSN : 1775-3546

\section{Éditeur}

Presses universitaires de Bordeaux

\section{Édition imprimée}

Date de publication : 1 novembre 1997

ISSN : 1168-5549

\section{Référence électronique}

Hugues Hotier, "Propos liminaire pour introduire sans trop induire », Communication et organisation [En ligne], 12 | 1997, mis en ligne le 26 mars 2012, consulté le 04 mai 2019. URL : http://

journals.openedition.org/communicationorganisation/1978; DOI : 10.4000/ communicationorganisation. 1978

Ce document a été généré automatiquement le 4 mai 2019.

(c) Presses universitaires de Bordeaux 


\title{
Propos liminaire pour introduire sans trop induire
}

\author{
Hugues Hotier
}

1 Chacun connaît cette technique d'investigation à laquelle on recourt pour établir le champ sémantique d'un mot dans une société donnée à une époque donnée. À un individu on envoie un substantif comme un stimulus et on recueille les réponses que l'audition de ce substantif génère. Je me souviens m'être amusé à ce jeu sérieux quand j'exerçais à Lille et que les travailleurs sociaux constataient avec surprise que les personnes qu'ils aidaient n'aimaient pas être appelés « clients». C'est ainsi que la psychologie rogérienne voulait qu'on dénommât ceux qui, jusque-là, n'avaient été que des "cas sociaux». Pour l'anecdote, nous constatâmes que client était un mot connoté négativement quand assistante sociale l'était positivement. Il était difficile à ces personnes de supporter qu'on les traitât si mal et qu'on ne leur retournât pas le respect dont elles-mêmes gratifiaient les travailleurs sociaux.

\section{Les deux acceptions du mot induction}

2 Si l'on applique la même technique au mot induction dans les usages qu'en font les spécialistes des sciences humaines, on constate que le champ sémantique s'organise autour de deux axes. C'est d'abord, et presque par réflexe, l'influence que le mot induction évoque et l'étymologie n'y est pas pour rien [latin inducere : conduire dans]. C'est ensuite, moins immédiat, moins direct et découlant d'une réflexion plus approfondie, tout ce qui concourt à la communication sans être pour autant lié au schéma canonique émetteur-message-récepteur. Et là, les mots manquant, on risquerait, si l'on n'y prenait garde, de tomber dans le lexique de l'ésotérisme. On parlerait de charme, de mystère, voire d'alchimie. Nous nous efforcerons au cours de ce colloque de ne point nous laisser aller et, raisonnant en chercheur plutôt qu'en zélateur ou en contempteur, nous tenterons d'éviter ces mots magiques dont la puissance d'évocation masque le vide sémantique et dont l'usage disqualifie la recherche. 


\section{Le processus d'influence}

3 démarche d'induction. On peut influencer volontairement ou malgré soi. Cette femme aussi intelligente et spirituelle que belle n'est pas obligatoirement responsable des modifications comportementales que sa présence génère chez moi. Quand l'influence est volontaire, elle peut appartenir à un véritable projet. Faut-il rappeler le projet politique des dictateurs que l'histoire et l'actualité offrent à nos regards consternés ? L'appellation duce qu'avait choisie l'un d'entre eux nous rappelle l'étymologie dont il était question il y a un instant. Sans aller jusque-là, Gino Gramaccia et Gérard Massard ${ }^{2}$ nous remémorent que, lorsqu'un manager induit volontairement ses collaborateurs, il s'agit pour lui d'« inscrire le sujet potentiel dans une logique de mission». C'est ce qu'on pourrait appeler l'induction pour le faire faire et, ici, l'induction ressortit à la pragmatique de la communication.

5 Voilà une première distinction qui se situe au niveau de l'émetteur. Il en est une autre qui doit s'opérer au niveau du récepteur/destinataire. Je suggère de parler de destinataire quand l'émetteur a une intentionnalité, quand la communication s'inscrit dans un projet, quand elle se fixe des objectifs à atteindre. Bien entendu, l'émetteur souhaite que le destinataire devienne récepteur. Mais il ne dépend pas que de lui que cette mutation s'opère: ce n'est qu'un souhait au service duquel il devra sans doute mettre toute sa technique, sinon tout son art. De même qu'on peut être émetteur et inducteur volontairement ou malgré soi, on peut distinguer trois types de récepteurs/destinataires ${ }^{3}$.

Je parlerai de destinataire disponible pour désigner le destinataire qui accepte de devenir récepteur sans pour autant participer au processus de transformation. "Informez-moi, instruisez-moi, convainquez-moi, séduisez-moi... mais ne comptez pas sur moi pour faire le premier pas. » semble-t-il dire. Ce destinataire-là ne demande qu'à devenir récepteur. Le destinataire coopératif va plus loin. Il participe à sa propre transformation, il contribue activement à l'établissement et à l'entretien de la communication. Enfin, le destinataire hostile s'oppose à sa propre mise en réceptivité. Ce qui, éventuellement, ne l'empêchera pas de devenir récepteur malgré lui. Soit parce que sa résistance est insuffisante pour s'opposer à l'émetteur, soit parce que cette résistance est contournée par une manipulation dont il n'a pas conscience.

7 Bruno Ollivier ${ }^{4}$ imagine que, pour se soumettre à l'induction, "le destinataire doit s'y retrouver " et il énonce » les cinq règles pour induire "Je ne résiste pas au plaisir de les rappeler ici en précisant que, nonobstant la forme malicieuse de l'énoncé, il ne s'agit pas d'un vademecum de l'apprenti manipulateur mais bien d'un constat de recherche :

«Pour transformer un destinataire en récepteur, il faudra ainsi faire en sorte :

$1 / q u$ 'il se sente reconnu

$10 \quad 2 / q u$ 'il se sente chez lui

$113 / q u ' i l$ y trouve son intérêt

$124 / q u$ 'il y trouve son plaisir

13

5/qu'il ne perde pas la face» 

que processus d'influence.

\section{Hors du schéma émetteur-récepteur}

Un ensemble complexe à processus multidimensionnels, interactifs, sans polarité prédictible, à l'œuvre dans la communication.

Ainsi avons-nous provisoirement défini l'induction quand nous ne la considérons pas comme une des formes de l'influence.

Dans une certaine mesure, une telle approche rejoint Ray Birdwhistell quand il écrit :

"Un individu ne communique pas; il prend part à une communication ou il en devient un élément. Il peut bouger, faire du bruit... mais il ne communique pas. Il peut entendre, sentir, goûter et toucher, mais il ne communique pas. En d'autres termes, il n'est pas auteur de la communication, il y participe». (cité par Watzlawick et allii dans Une logique de la communication, Paris, Seuil, 1972, page 68).

17 Il peut sembler incongru de parler de communication sans émetteur. Et pourtant, nous avons évoqué dans les Textes préparatoires des situations dans lesquelles un environnement conçu pour une finalité précise produit des effets tout à fait autres que ceux qui sont attendus. L'odeur de confiserie qui flotte aux alentours du cirque est produite pour attirer le chaland et n'est pas faite pour mettre en réceptivité le futur spectateur qui s'avance vers le chapiteau. L'émetteur de cette odeur n'imagine pas pouvoir être autre chose qu'un confiseur. Cependant, c'est essentiellement cette mise en réceptivité, ce conditionnement du spectateur que l'odeur et son émetteur produisent. En ce sens, on pourrait effectivement dire avec Birdwhistell que le marchand de confiserie ne communique pas et que le futur spectateur qui perçoit les effluves des barbes à papa et autres pommes d'amour ne communique pas. Tous deux entrent dans un processus de communication en tant que participants mais on ne peut, à proprement parler, dire qu'ils sont les auteurs d'une communication.

Valérie Carayol ${ }^{5}$ rappelle qu'Umberto Eco parle de processus de communication dans lesquels il n'existe pas de code et qu'il assimile à des processus de type stimulus-réponse. Et elle est ainsi amenée à se poser la question de l'existence de processus infrasémiotiques non assimilables à des stimuli. Tout aussi passionnantes à étudier sont ces formes de communication auxquelles recourt le marketing et qui ont pour objet de faire naître le désir de possession. Valérie Carayol rappelle que la SNCF travaille à la conception d'un « design sonore » pour les gares et rappelle que, pour pousser à l'achat, les croissanteries diffusent autour de leur boutique un parfum de viennoiserie parfaitement artificiel. Dans le même ordre d'idées, elle évoque encore la somesthésique, néologisme par lequel Lacina Traore désigne une forme de communication par le toucher, qui a pour objet ou pour effet de générer le désir. Le toucher peut être réalisé par la main mais aussi par d'autres parties du corps.

19 On aborde ici la communication par la sensorialité et même par son corollaire la sensualité. Allant plus loin que Roland Barthes et son célèbre «bruissement de la langue », Nicole Denoit parle de " la vibration charnelle dont procède l'induction. $~^{6}$

On a trop longtemps privilégié le signifié et considéré la communication comme un exercice intellectuel ayant pour objet prioritaire le transfert d'information. Souvenonsnous de la boutade de Roland Barthes déconseillant de dire deux fois Je t'aime à une femme car «la deuxième fois, il n'y a plus d'information». On sait bien que la 
communication est aussi, est surtout, relation. Et que cette relation est, pour une large part, fondée sur le plaisir : plaisir de la co-présence, plaisir du souvenir, plaisir de la reconnaissance, plaisir de natures et de formes les plus diverses.

21 Ce plaisir peut être des plus subtils. Il l'est assurément quand la communication confirme au récepteur-demandeur le bien-fondé de l'analyse ou du diagnostic qu'il avait lui-même formulé. Reprenant les travaux de l'ethnologue Jeanne Favret-Saada, Annie Gilles ${ }^{7}$ montre bien que ce que l'individu qui se croit ensorcelé vient chercher chez Madame Flora la voyante, c'est d'abord une confirmation de cet ensorcellement dont il a conscience ou dont un ancien ensorcelé lui a parlé. Certes, les ensorcelés sont rares dans notre société moderne mais on pourrait, avec Ghislaine Dolo-Thorion ${ }^{8}$, rappeler que les managers qui font appel aux consultants ont un comportement très voisin du leur. Eux aussi espèrent le plaisir de voir confirmer la sureté de leur diagnostic et ce sont « les signes de l'induction "qui permettent de repérer ce désir.

22 Ces signes de l'induction, Jean-Pierre Callegari' les repère dans une situation de communication qu'avec Michael Chekhov il interprète en termes de théâtralité comme "une improvisation collective, un échange ininterrompu entre les partenaires où chacun donne et reçoit à la fois. La plus légère indication de l'un d'eux (un coup d'œeil, un temps d'arrêt, une information imprévue, un geste, un soupir, ou même un changement de rythme à peine perceptible), doit suffire à inviter l'autre à lancer l'improvisation dans une nouvelle direction ”

\section{De la question de départ à un colloque qui n'est pas une ligne d'arrivée}

"Au départ d'une recherche ou d'un travail, le scénario est pratiquement toujours identique. On sait vaguement que l'on veut étudier tel ou tel problème... mais on ne voit pas très bien comment aborder la question. $»^{10}$

23 C'est pour rassurer les jeunes chercheurs débutants que Raymond Quivy et Luc Van Campenhoudt écrivent ces lignes dès les premières pages de leur excellent Manuel de recherche en sciences sociales. Les membres du GREC/O ne sont plus de jeunes chercheurs et le regrettent sans doute. Il n'empêche que quand on se fixe pour objet de comprendre la communication en s'efforçant de ne pas l'aborder par le schéma habituel émetteurrécepteur, quand on envisage de comprendre comment on participe à une communication plutôt qu'on ne communique, pour reprendre la formule de Birdwhistell, on est rassuré de savoir que l'angoisse qu'on ressent est quasi naturelle. Georges Devereux n'a-t-il pas intitulé l'un de ses ouvrages De l'angoisse à la méthode?

Autant le dire d'emblée, l'objet de cette recherche, je veux dire la compréhension de cet " ensemble complexe à processus multidimensionnels, interactifs, sans polarité prédictible «dont nous pressentons la réalité dans la communication a été pour nous anxiogène dans la mesure où nous ne savions par quel bout le prendre.

25 J'ai raconté dans les Textes préparatoires comment nous avons observé les situations de communication pour en extraire les tendances, les constantes, les invariants sinon les universaux. Comment ensuite, nous avons essayé de conceptualiser, de théoriser ces situations qui perdaient leur statut pour n'être plus que de simples illustrations de principes que nous énoncions avec la plus extrême prudence.

26 Ces Textes préparatoires qui sont entre les mains des participants au colloque ${ }^{11}$ font état de nos découvertes et de nos réflexions. Si nous avons organisé cette rencontre, c'est que 
nous voulions aller plus loin. Nous voulions, nous voulons aujourd'hui, entendre d'autres chercheurs réagir à nos propositions. C'est pourquoi nous les soumettons, en toute modestie, à votre examen.

$$
\begin{aligned}
& \text { avec les resp } \\
& \text { colloque, }
\end{aligned}
$$

34 


\section{NOTES}

1. Mesnil Christian «Induction et argumentation- in Induction et communication - Textes préparatoires. Bordeaux, GREC/O, 1997, p. 152.

2. Gramaccia Gino et Massard Gérard, "Induction, communication et management -Quatre hypothèses théoriques » in Induction et communication - Textes préparatoires, op. cit., p. 95.

3. Hotier Hugues, «L'emprise des sens » in Induction et communication - Textes préparatoires, op.cit., pp. 116-117.

4. Hotier Hugues, «L'emprise des sens » in Induction et communication - Textes préparatoires, op.cit., pp. 116-117.

5. Carayol Valérie, "Communication, induction et mimesis » in Induction et communication Textes préparatoires, op.cit., p. 39.

6. Denoit Nicole, "L'induction ou «le frisson magnétique du sens" » in Induction et communication - Textes préparatoires, op cit., p. 51.

7. Gille Annie, «L'induction ou les champs de force du désir » in Induction et communication Textes préparatoires, op.cit., p. 74.

8. Dolo-Thorion Ghyslaine, « Méthodologie d'approche du champ de l'induction dans le domaine de la communication » in Induction et communication -Textes préparatoires, op.cit., p. 61.

9. Callegari Jean-Pierre, "Induction et mise en scène de la communication » in Induction et communication - Textes préparatoires, op.cit., pp. 19-20.

10. Quivy Raymond et Van Campenhoudt Luc, Manuel de recherche en sciences sociales, Paris, Dunod, $2^{\mathrm{e}}$ édition, 1995 , p. 9

11. On peut se les procurer au GREC/O Institut des Sciences de l'information et de la Communication, Université Michel de Montaigne-Bordeaux 3, Domaine universitaire 33405 Talence Cedex. 